\title{
UKIDSS detections of cool brown dwarfs
}

\section{Proper motions of 14 known $>$ T5 dwarfs and discovery of three new T5.5-T6 dwarfs $\star, \star \star$ (Research Note)}

\author{
R.-D. Scholz, G. Bihain, O. Schnurr, and J. Storm
}

Leibniz-Institut für Astrophysik Potsdam (AIP), An der Sternwarte 16, 14482 Potsdam, Germany

e-mail: [rdscholz; gbihain; oschnurr; jstorm]@aip.de

Received 2 February 2012 / Accepted 8 April 2012

\section{ABSTRACT}

\begin{abstract}
Aims. We contribute to improving the census of cool brown dwarfs (late-T and Y dwarfs) in the immediate solar neighbourhood. Methods. By combining near-infrared (NIR) data of UKIDSS with mid-infrared WISE and other available NIR (2MASS) and red optical (SDSS $z$-band) multi-epoch data we detected high proper motion (HPM) objects with colours typical of late spectral types (>T5). We used NIR low-resolution spectroscopy for the classification of new candidates.

Results. We determined new proper motions for 14 known T5.5-Y0 dwarfs, many of which are significantly ( $>2-10$ times) more accurate than previous ones. We detected three new candidates, ULAS J0954+0623, ULAS J1152+0359, and ULAS J1204-0150, by their HPMs and colours. Using previously published and new UKIDSS positions of the known nearby T8 dwarf WISE J0254+0223 we improved its trigonometric parallax to $165 \pm 20$ mas. For the three new objects we obtained NIR spectroscopic follow-up with LBT/LUCIFER classifying them as T5.5 and T6 dwarfs. With their estimated spectroscopic distances of about 25-30 pc, their proper motions of about 430-650 mas/yr lead to tangential velocities of about $50-80 \mathrm{~km} \mathrm{~s}^{-1}$, typical of the Galactic thin-disk population.
\end{abstract}

Key words. astrometry - proper motions - stars: distances - stars: kinematics and dynamics - brown dwarfs - solar neighborhood

\section{Introduction}

Although brown dwarfs (BDs) might be even more numerous than M-type red dwarf stars, to date we know about ten times less substellar objects than stars in the immediate solar neighbourhood (within $\sim 6 \mathrm{pc}$ ). The BDs are difficult to detect, especially in the optical, because of their relatively low fluxes and red opticalto-near-infrared colours. This is reinforced by (i) their "failedstar" nature, because they dim and cool down with age, evolving across the M, L, and T spectral types (Burrows et al. 2001), and (ii) the typical age of several Gyr of solar neighbourhood objects. Most of the BD neighbours are expected to have reached temperatures as low or lower than those of T dwarfs ( 500-1300 K), which is confirmed by the fact that almost $75 \%$ of the objects with trigonometric parallaxes $>100$ mas in the compilation of $\mathrm{L}$ and T dwarfs of Gelino et al. (2011, hereafter G11) are T dwarfs, and their fraction continues to grow, in particular with late-T (>T5) types.

Deep large-area optical surveys like the Sloan Digital Sky Survey data releases (SDSS DR7, Abazajian et al. 2009; DR8, Aihara et al. 2011) detected only very nearby late-T dwarfs. The Canada-France BD survey is deeper, although smaller in sky area (Delorme et al. 2008, hereafter D08; Albert et al. 2011). New near-infrared (NIR) and mid-infrared surveys covering big sky portions such as the Large Area Survey (LAS) within the

\footnotetext{
* Based on observations with the Large Binocular Telescope (LBT)

$\star \star$ Tables $1-5$ are available in electronic form at http://www . aanda.org
}

UKIRT InfraRed Deep Sky Surveys (UKIDSS) ${ }^{1}$, which goes deeper than the Two Micron All Sky Survey (2MASS; Skrutskie et al. 2006), and the Wide-field Infrared Survey Explorer (WISE; Wright et al. 2010), which extends the 2MASS to the midinfrared over the whole sky, are very sensitive to late-T dwarfs and even cooler Y dwarfs, as recently shown by Kirkpatrick et al. (2011, hereafter K11) and Cushing et al. (2011, herafter C11).

In continuation of the study of Scholz (2010, hereafter S10), who used UKIDSS DR6 and SDSS DR7 to detect late-T dwarf candidates, and Scholz et al. (2011, hereafter S11), who combined the WISE preliminary data release (PDR) with SDSS (DR7+DR8) and 2MASS discovering two very nearby ultracool BDs and classifying one of them (WISE J1741+2553) spectroscopically as a T9.5 dwarf, we looked for more late-T dwarf candidates with HPMs. We combined recent UKIDSS data releases (DR7+DR8+DR9) with WISE, 2MASS, and SDSS, where available. The UKIDSS releases represent a growing database, whereas SDSS DR7 and DR8 data are independent and may differ for a given object. Sections 3 and 5 provide proper motions and photometry of 14 previously known and three newly found objects, respectively. Section 4 provides the parallax measurement of a very nearby object. Section 6 presents

\footnotetext{
1 The UKIDSS project is defined in Lawrence et al. (2007). UKIDSS uses the UKIRT Wide Field Camera (WFCAM; Casali et al. 2007) and a photometric system described in Hewett et al. (2006), which is situated in the Mauna Kea Observatories (MKO) system (Tokunaga et al. 2002). The pipeline processing and science archive are described in Hambly et al. (2008) and Irwin et al. (in prep.).
} 
spectroscopic follow-up for our new candidates, and Sect. 7 contains our brief discussion and conclusions.

\section{Selection of candidates and cross-identification}

Nearby cool BDs are expected to be very faint HPM objects. We identify them not only based on their characteristic colours in one deep survey but as moving objects showing up in various surveys with time baselines of several years. For our search of nearby $>\mathrm{T} 5$ dwarfs, primarily based on latest UKIDSS, i.e. DR9 LAS and Galactic Clusters Survey (GCS), and SDSS data, we used the same criteria as described in S10:

$J>11, \quad J-K<0, \quad z-J>+2.5$.

Only late-T dwarfs and possibly low-metallicity $\mathrm{T}$ dwarfs meet these constraints. White dwarfs, late-M and L dwarfs are excluded by the two colour cuts. To identify an HPM object, we needed at least two different epochs from UKIDSS or other available survey data, if it had no suitable SDSS counterpart. For additional survey data we looked at 2MASS and other catalogues accessible via the $\mathrm{CDS}^{2}$. We also checked 2MASS FITS images from the IRSA Finder $\mathrm{Chart}^{3}$ and detected objects visually using "pick object" within the ESO skycat tool. After the WISE PDR, we checked our UKIDSS candidates, including those with lacking/uncertain proper motions, for WISE counterparts. In the WISE PDR, we also selected suspected HPM objects (no 2MASS counterpart within 3 arcsec) with late-T dwarf mid-infrared colours ${ }^{4}$ (similar to the criteria used by S11):

$w 1-w 2>+1.5, \quad w 2-w 3<+2.5, \quad w 2<15$,

and subsequently looked for UKIDSS counterparts. However, using WISE as a starting point, we found only already known objects. We checked the UKIDSS DR9 LAS, GCS, and DR7 Galactic Plane Survey (GPS) for possible measurements of the > T5 dwarfs discovered by K11, to improve their proper motions by involving additional epochs. UKIDSS counterpart data were found in the LAS and GCS but not in the GPS. In Sects. 3 and 5, we show our proper motion results for objects with at least three distinct epochs available.

\section{Proper motions and photometry of known objects}

In Table 1 we list the photometry and our proper motions of previously known late-T (>T5) dwarfs, which we newly identified in UKIDSS LAS data. Individual multi-epoch positions from UKIDSS LAS (and other surveys) are listed in Tables 2-4, where our visual 2MASS detections are marked. Imaging SDSS and UKIDSS LAS data are available in all fields/bands of the ten objects. For objects not detected in any of the bands listed in Table 1, we give lower magnitude limits corresponding to the SDSS depths (Abazajian et al. 2009) and in specific fields of UKIDSS LAS and GCS (using SQL queries from Dye et al. 2006). Most of the LAS detections are recent WISE discoveries (K11). Full designations of all known objects described in this paper can be found in K11 and G11.

We also aimed to improve the proper motions for three known > T5 dwarfs from Table 6 of S10 (not shown here again)

\footnotetext{
2 http://vizier.u-strasbg.fr/cgi-bin/VizieR

3 http://irsa.ipac.caltech.edu/applications/ FinderChart/

$4 w 1, w 2$, and $w 3$ bands are centered at $3.4 \mu \mathrm{m}, 4.6 \mu \mathrm{m}$, and $12 \mu \mathrm{m}$
}

by involving WISE positions if available. However, this led to larger uncertainties in $\mu_{\delta}$. In particular, we note that one of these three (the T7 dwarf SDSS J1507+1027; Chiu et al. 2006) is detected by WISE as a red object $(w 1=16.30 \pm 0.09$, $w 2=14.12 \pm 0.05, w 3=12.13 \pm 0.33$ ) but shifted in declination by about 1.5 arcsec with respect to its expected position according to the HPM measurement of S10. According to the WISE PDR documentation, sources fainter than $w 1 \sim 14.5$ may have "declinations being offset between 0.2 and 1.0 arcsec from the true position". This object is not included in K11. The T6.5 dwarf SDSS J1346-0031 (Tsvetanov et al. 2000; Burgasser et al. 2006) in Table 1 is also a red WISE source $(w 1=15.41 \pm$ $0.05, w 2=13.56 \pm 0.04, w 3=11.99 \pm 0.32$ ) but was not listed by K11 in their Table 1 (with WISE and NIR photometry of known T dwarfs).

We determined the new proper motions in Table 1 from weighted linear fitting over all available multi-epoch positions, including 2MASS and SDSS (assuming 100 mas errors), WISE PDR (with their given errors), and UKIDSS positions (assuming 70 mas errors) listed in Tables 2-4, as well as all WISE and Spitzer measurements (with their errors) given in K11 if available. In Sect. 4, we compare our error assumptions with others. For faint UKIDSS ( $>18$ mag) objects we assumed larger errors (140 mas), and for faint SDSS $(z>20)$ objects and our visual 2MASS detections, we increased the assumed errors to 150 mas and 200 mas, respectively. For CFBDS J0059-0114 we included the CFBDS position (assuming 150 mas errors) from D08 in our proper motion fitting. For WISE J0254+0223, we combined 2MASS and SDSS DR8 positions already used by S11 with PanSTARRS1 measurements from Liu et al. (2011, hereafter L11), multiple WISE (instead of WISE PDR) and Spitzer epochs provided by K11, and UKIDSS DR9 data. We improved the trigonometric parallax for the latter object (see Sect. 4).

Our proper motions of the first six WISE discoveries in Table 1 are at least 2-5 times more accurate than previous values in S11 and K11, whereas those of CFBDS J0059-0114, ULAS J0827-0204 and SDSS J1346-0031 agree with more accurate other data. Pinfield et al. (2012) measured the proper motion of WISE J0750+2725 using only two UKIDSS epochs. By involving the WISE and Spitzer epochs, we obtained a smaller $\mu_{\alpha} \cos \delta$, whereas our $\mu_{\delta}$, despite a poor fit, agrees well with the former value. For SDSS J1110+0116 our $\sim 2 \times$ more accurate result agrees well with that of Jameson et al. (2008), who did not use the SDSS.

Because WISE J0750+2725 was not detected in SDSS z-, UKIDSS $H K$-bands, and 2MASS, the maximum epoch difference between the first epoch UKIDSS $J$-band and the last epoch Spitzer data was less than four years. The resulting proper motion errors are relatively large, but the total proper motion is highly significant. Even larger errors for CFBDS J0059-0114, WISE J2226+0440 and WISE J2344+1034 are caused by maximum epoch differences of only about 1.5 to 2 years. The worst case is WISE J1311+0122, where the UKIDSS data do not significantly extend the time baseline of only $\sim 1$ year, resulting in the largest proper motion errors. However, all large proper motion components, already indicated in K11, were confirmed.

For four of the > T5 discoveries of K11, we found UKIDSS GCS $K$-band data (Table 1 ). However, one of the Y0 dwarfs was not detected therein. For the others we used the positions of their apparent counterparts (Table 3) together with 2010/2011 WISE and Spitzer epochs given in K11 for new proper motion solutions. For the T6 dwarf WISE J0410+1411, we included available SDSS DR8 data as well. We also found the known lateT dwarf 2MASS J0516-0445 in our search combining WISE 


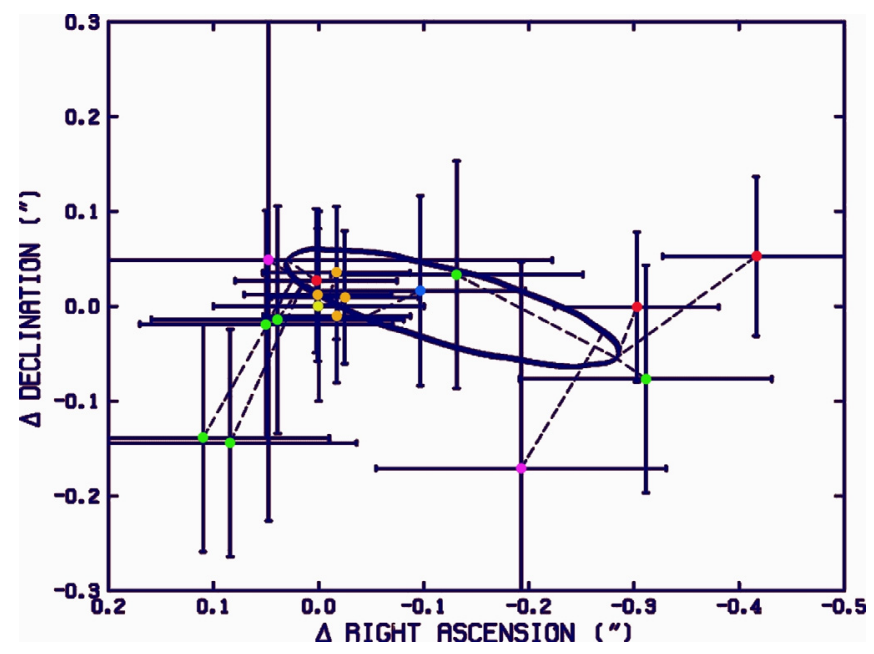

Fig. 1. Parallactic ellipse for WISE J0254+0223 after combined proper motion and parallax solution and subtraction of the proper motion. Coloured (blue - SDSS, green - Pan-STARRS1, yellow - 2MASS, orange - UKIDSS, red - WISE, pink - Spitzer) dots with error bars show the individual positional measurements, dashed lines show their displacements relative to the fit.

and UKIDSS GCS data. We achieved $\sim 3 \times$ to $>10 \times$ more accurate proper motions for the T dwarfs with GCS detection (Table 1) but not for the Y0 dwarf WISE J1541-2250, which lies in a relatively crowded region close to Upper Sco (Lodieu et al. 2008) and has highly discrepant preliminary trigonometric $(2-4 \mathrm{pc})$ and photometric $(\sim 8 \mathrm{pc})$ distances in K11. If the latter is correct, we would not expect to see a counterpart in the GCS $K$-band, similar to what we found for the other Y0 dwarf, WISE J0410+1502, which has according to K11 a photometric distance of $\sim 9 \mathrm{pc}$. Therefore, we think the GCS counterpart of WISE J1541-2250 and its revised proper motion are doubtful.

\section{Trigonometric parallax for WISE J0254+0223}

Preliminary trigonometric parallaxes for WISE J0254+0223, which has the largest proper motion in Table 1, were published by L11 and K11. By combining all available measurements used by these authors (less than 10 epochs in both cases) with new UKIDSS DR9 data (Table 2), we collected 17 epochs and expected an improved fit. We applied the software of Gudehus (2001) for combined proper motion and parallax solutions with and without weights corresponding to the positional errors of the different surveys. In our final weighted solution, which yielded $\sim 10 \%$ more accurate parallax and proper motion results compared to the unweighted one, we used the following errors: 70 mas for UKIDSS (which we can assume for an object at intermediate galactic latitude according to Lawrence et al. 2007), 100 mas for 2MASS and SDSS, 120 mas for PanSTARRS1 (corresponding to the typical size of the error bars shown in Fig. 4 of L11, and the individual errors of the three WISE (between 76 mas and 89 mas) and two Spitzer epochs (from 138 mas to 275 mas) given in K11. Our assumed 2MASS errors are smaller than the values given in K11 and correspond to those of L11. For the SDSS counterpart we assumed slightly larger errors than L11 because we took into account that the astrometric accuracy at the faint end of the SDSS is limited by photon statistics (Pier et al. 2003). Our proper motion and parallax of WISE J0254+0223 are

$\left(\mu_{\alpha} \cos \delta, \mu_{\delta}\right)=(+2544 \pm 7,+237 \pm 7)$ mas $/ y r, \pi=165 \pm 20$ mas.
The proper motion is more accurate than that in Table 1 from linear fitting not taking into account the parallactic motion. In Fig. 1 we show the parallactic ellipse and the individual positions with their error bars and with respect to their expected location (indicated by dashed lines) according to the fit after subtracting the proper motion. Only in four cases the shift between expected location and measured position exceeds the error bars. This concerns three out of six Pan-STARRS1 and one out of three WISE positions, whose errors may be underestimated.

The distance estimate of $6.1 \pm 0.7 \mathrm{pc}$ from the new parallax agrees well with the photometric distances of $5.5_{-1.6}^{+2.3} \mathrm{pc}$ (S11), $7.2 \pm 0.7 \mathrm{pc}$ (L11), and $\sim 6.9 \mathrm{pc}$ (K11). Our new parallax is also very similar to previous trigonometric measurements of $171 \pm 45$ mas (from nine positions) and $165 \pm 46$ mas (from six positions) by L11 and K11, respectively, but has a 50\% smaller error. However, our new parallax is still a preliminary result, not obtained within a dedicated parallax programme and making use of different optical, NIR, and mid-infrared surveys. Because we neglected colour-dependent systematic errors in the positions (differential refraction), our parallax errors are lower limits. We also derived significant parallaxes for ULAS J0827-0204 (97 \pm 21 mas), SDSS J1110+0116 (56 \pm 5 mas), and the new T5.5 dwarf (Sects. 5 and 6) ULAS J0954+0623 (136 \pm 28 mas), but these results are uncertain (from only six epochs in each case).

\section{New late-T dwarf candidates}

Using the UKIDSS LAS as a starting point, we discovered three new late-T dwarf candidates: ULAS J095429.91+062309.9, ULAS J115229.67+035927.2, and ULAS J120444.67-015034.8 (hereafter names are abbreviated). They have $z$-only SDSS counterparts shifted by several arcsec with respect to their two LAS epochs. For our proper motion fits we measured their positions at a fourth (earliest) epoch by visually detecting 2MASS counterparts (Table 4). The resulting well-measured HPMs, and the $z Y J H K$ magnitudes of the objects are shown in Table 1. No WISE PDR data are available for these objects.

All three new candidates can be preliminarily classified as $\mathrm{T} 7 \pm 2$ dwarfs based on their negative NIR colours $-0.41<J-H<-0.26$ and $-0.58<J-K<-0.48$. As shown by $\mathrm{S} 10$, this colour-based classification cannot be made more accurately for this range of negative NIR colour indices. This is due to the colour reversal of T8-T9 dwarfs, whereas the sequence of T dwarfs with earlier types shows a clear trend with both colours, which allows a more accurate colour-based classification for positive and moderately negative indices (see Fig. 3 in S10). The more accurate spectral types (SpT) given in Table 1 are the result of our spectroscopic follow-up described in the next section.

\section{Spectroscopic observations with LBT/LUCIFER}

For follow-up we used the Large Binocular Telescope (LBT) NIR spectrograph LUCIFER1 (Mandel et al. 2008; Seifert et al. 2010; Ageorges et al. 2010) in long-slit spectroscopic mode with the $H+K$ (200 lines/mm + order separation filter) and $z J H K$ gratings (210 lines/mm $+J$ filter). ULAS J1204-0150 and ULAS J0954+0623 were observed with total integrations of $30 \mathrm{~min}$ in $H+K$ and $20 \mathrm{~min}$ in $J$ on 11 April 2011 and 12 April 2011, respectively. The slighly fainter ULAS J1152+0359 was observed on 11 May 2011 with $36 \mathrm{~min}$ in $H+K$ and $24 \mathrm{~min}$ in $J$. As in S11, central wavelengths were chosen at $1.835 \mu \mathrm{m}$ 

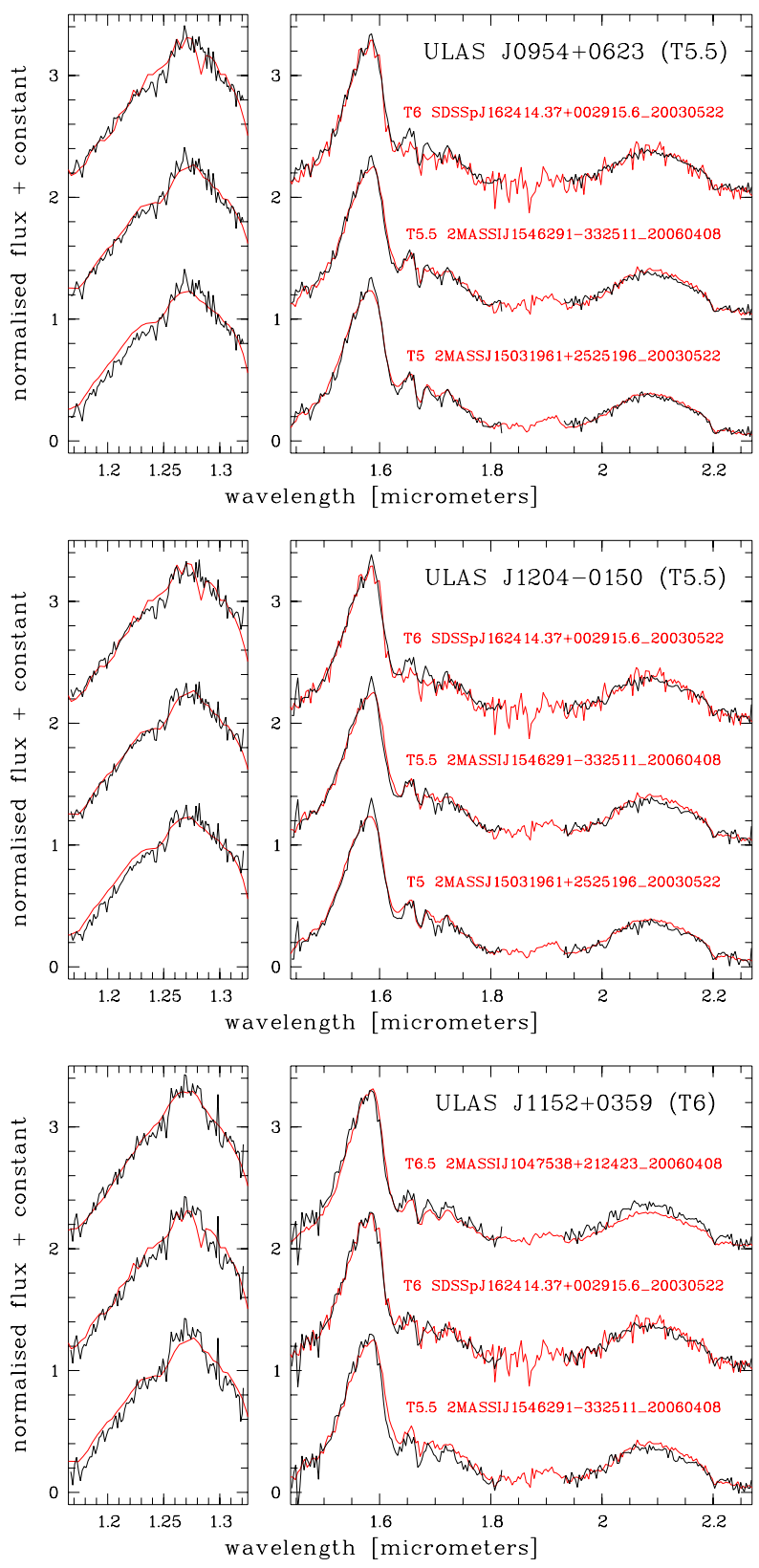

Fig. 2. LBT/LUCIFER1 $J$ - (left) and $H+K$-band (right) spectra of ULAS J0954+0623 (top), ULAS J1204-0150 (centre), and ULAS J1152+0359 (bottom). Target spectra (black) were smoothed for the comparison with the lower-resolution $(R \sim 120)$ template spectra (red) of T5-T6.5 dwarfs from Adam Burgasser's SpeX Prism Spectral Libraries. Original references for the T5/T6 and T5.5/T6.5 template spectra are Burgasser et al. (2004) and Burgasser et al. (2008), respectively.

$(H+K)$ and $1.25 \mu \mathrm{m}(J)$ yielding a coverage of 1.38-2.26 and $1.17-1.32 \mu \mathrm{m}$, respectively. The slit width was 1 arcsec for ULAS J1152+0359, corresponding to 4 pixels, with a spectral resolving power $R=\lambda / \Delta \lambda \approx 4230,940$, and 1290 at $\lambda \approx 1.24$, 1.65 , and $2.2 \mu \mathrm{m}$, respectively. For the other objects the slit width was 2 arcsec. Observations consisted of individual exposures of $75 \mathrm{~s}$ in $H+K$ and $200 \mathrm{~s}$ in $J$ (90 and $240 \mathrm{~s}$, respectively, for ULAS J1152+0359) with shifting the target along the slit using an ABCCBA pattern until the total integration time was reached. A0V standards were observed just before/after the targets with similar airmasses.
The raw spectroscopic data were reduced as described in S11 using standard routines in $\operatorname{IRAF}^{5}$. The calibrated $J$ and $H+K$ spectra of our new objects and the corresponding spectra of the templates shown in Fig. 2 were normalised by the average flux in the range $1.20-1.30$ and $1.52-1.61 \mu \mathrm{m}$, respectively. Each spectrum was classified by visual comparison with template spectra, separately for the $J$ - and $H+K$-band, and by three measured spectral indices in the $H+K$-band (Table 5) as defined by Burgasser et al. (2006). No other useful indices could be determined in the $J$-band, since the LUCIFER $J$ grating provides a very narrow wavelength interval. Figure 2 shows the three closest matches of template (in red) with smoothed target spectra (overplotted in black). Both methods, comparison with templates and classification with indices, provided consistent results (with \pm 0.5 sub-types accuracy, as in S11): ULAS J0954+0623 and ULAS J1204-0150 were classified as T5.5, whereas ULAS J1152+0359 turned out to be a T6 dwarf.

Using the relationship between spectral types and absolute JHK magnitudes determined by Marocco et al. (2010) from L0-T9 dwarfs with known trigonometric parallaxes (excluding known and possible binaries), we obtain $M_{J}=14.70, M_{H}=$ 14.92, $M_{K}=14.99$ for T5.5 and $M_{J}=14.92, M_{H}=15.22$, $M_{K}=15.32$ for T6 dwarfs. Assuming conservatively absolute magnitude uncertainties of about $\pm 0.4 \mathrm{mag}$, involving our \pm 0.5 sub-type classification, we obtain spectroscopic distances of $25 \pm 5$ pc, $31 \pm 6$ pc, and $27 \pm 6$ pc for ULAS J0954+0623, ULAS J1152+0359, and ULAS J1204-0150, respectively.

\section{Discussion and conclusions}

Searching HPM > T5 dwarfs in the latest UKIDSS LAS/GCS data we detected some previously known objects, including nine recent WISE discoveries (K11), and revised their proper motions. For the very nearby T8 dwarf WISE J0254+0223 (S11, L11, K11) we derived an improved trigonometric parallax. The coolest known objects in our sample, two recently classified Y0 dwarfs $(\mathrm{C} 11)$, were either too faint to be detected or have a doubtful counterpart in the UKIDSS GCS.

We found new late-T candidates in the UKIDSS LAS and confirmed their >T5 types by LBT/LUCIFER1 NIR spectroscopy. ULAS J0954+0623, ULAS J1152+0359, and ULAS J1204-0150 are classified as T5.5, T6, and T5.5 dwarfs, respectively, all residing within $\sim 30 \mathrm{pc}$ from the sun. From their total proper motions of 650,441 , and 432 mas/yr, we computed tangential velocities of $77 \pm 15,65 \pm 13$, and $55 \pm 12 \mathrm{~km} \mathrm{~s}^{-1}$, respectively. These values are lower than the $87 \pm 8 \mathrm{~km} \mathrm{~s}^{-1}$ of WISE J0254+0223, for which L11 found thin-disk membership. We conclude that the new nearby cool BDs also belong kinematically to the thin-disk population of the Galaxy.

More accurate proper motions allow a more reliable determination of companionship to nearby stars. We checked the previously known objects (with our new proper motions) and our newly discovered ones for possible wide companions with common proper motions in SIMBAD $^{6}$ or in the database on $\mathrm{L}$ and $\mathrm{T}$ dwarfs provided by $\mathrm{G} 11$. Using a search radius of one degree around the target positions, we found no bright or faint wide companion candidate for which the proper motion agrees within the error bars and the projected separation is smaller than about

5 IRAF is distributed by the National Optical Astronomy Observatories, which are operated by the Association of Universities for Research in Astronomy, Inc., under cooperative agreement with the National Science Foundation.

6 http://simbad.u-strasbg.fr 
$10000 \mathrm{AU}$, if our estimated (or known) distances are taken into acount.

With our HPM- and colour-based survey, we have added three relatively nearby objects to the pool of $\sim 40$ previous UKIDSS discoveries of cool (>T5) BDs (Warren et al. (2007; Lodieu et al. 2007; Chiu et al. 2008; Pinfield et al. 2008; Lodieu et al. 2009; Lucas et al. 2010; Burningham et al. 2010). Most of them were identified based on their colours without proper motion measurements. Our three new objects are among the five brightest (in $J$ ) of all > T5 UKIDSS discoveries.

Acknowledgements. The authors thank Roland Gredel, Jochen Heidt, Jaron Kurk, Ric Davies, and all observers at the LBT for assistance during the preparation and execution of LUCIFER observations, and Adam Burgasser for providing template spectra at http://pono.ucsd.edu/ adam/browndwarfs/ spexprism. This research has made use of the WFCAM Science Archive providing UKIDSS, the NASA/IPAC Infrared Science Archive, which is operated by the Jet Propulsion Laboratory, California Institute of Technology, under contract with the National Aeronautics and Space Administration, and of data products from WISE, which is a joint project of the University of California, Los Angeles, and the Jet Propulsion Laboratory/California Institute of Technology, funded by the National Aeronautics and Space Administration, from 2MASS, and from SDSS DR7 and DR8. Funding for SDSS-III has been provided by the Alfred P. Sloan Foundation, the Participating Institutions, the National Science Foundation, and the US Department of Energy. The SDSS-III web site is http://www.sdss3.org/. We used SIMBAD and VizieR at the CDS/Strasbourg.

\section{References}

Abazajian, K. N., Adelman-McCarthy, J. K., Agüeros, M. A., et al. 2009, ApJS, 182,54

Ageorges, N., Seifert, W., Jütte, M., et al. 2010, SPIE, 7735, 53

Aihara, H., Allende Prieto, C., An, D., et al. 2011, ApJS, 193, 29

Albert, L., Artigau, É., Delorme, P., et al. 2011, AJ, 141, 203

Burgasser, A. J., McElwain, M. W., \& Kirkpatrick, J. D. 2003, AJ, 126, 2487

Burgasser, A. J., McElwain, M. W., Kirkpatrick, J. D., et al. 2004, AJ, 127, 2856

Burgasser, A. J., Geballe, T. R., Leggett, S. K., Kirkpatrick, J. D., \& Golimowski, D. A. 2006, ApJ, 637, 1067

Burgasser, A. J., Liu, M. C., Ireland, M. J., Cruz, K. L., \& Dupuy, T. J. 2008, ApJ, 681, 579
Burningham, B., Pinfield, D. J., \& Lucas, P. W. 2010, MNRAS, 410, 1885 Burrows, A., Hubbard, W. B., Lunine, J. I., \& Liebert, J. 2001, Rev. Mod. Phys., 73, 719

Casali, M., Adamson, A., Alves de Oliveira, C., et al. 2007, A\&A, 467, 777

Chiu, K., Fan, X., Leggett, S. K., et al. 2006, AJ, 131, 2722

Chiu, K., Liu, M. C., Jiang, L., et al. 2008, MNRAS, 385, L53

Cushing, M. C., Kirkpatrick, J. D., Gelino, C. R., et al. 2011, ApJ, 743, 50 (C11)

Delorme, P., Delfosse, X., Albert, L., et al. 2008, A\&A, 482, 961 (D08)

Dye, S., Warren, S. J., Hambly, N. C., et al. 2006, MNRAS, 372, 1227

Faherty, J. K., Burgasser, A. J., Cruz, K. L., et al. 2009, AJ, 137, 1

Geballe, T. R., Knapp, G. R., Leggett, S. K., et al. 2002, ApJ, 564, 466

Gelino, C. R., Kirkpatrick, J. D., \& Burgasser, A. J. 2011, online database for $804 \mathrm{~L}$ and T dwarfs at DwarfArchives.org (status: 14 February, 2011) (G11) Gudehus, D. H. 2001, BAAS, 33, 850

Hambly, N. C., Collins, R. S., Cross, N. J. G., et al. 2008, MNRAS, 384, 637

Hewett, P. C., Warren, S. J., Leggett, S. K., \& Hodgkin, S. T. 2006, MNRAS, 367,454

Jameson, R. F., Casewell, S. L., Bannister, N. P., et al. 2008, MNRAS, 384, 1399 Kirkpatrick, J. D., Cushing, M. C., Gelino, C. R., et al. 2011, ApJSS, 197, 19 (K11)

Lawrence, A., Warren, S. J., Almaini, O., et al. 2007, MNRAS, 379, 1599

Liu, M. C., Deacon, N. R., Magnier, E. A., et al. 2011, ApJ, 740, L32 (L11)

Lodieu, N., Pinfield, D. J., Leggett, S. K., et al. 2007, MNRAS, 379, 1423

Lodieu, N., Hambly, N. C., Jameson, R. F., \& Hodgkin, S. T. 2008, MNRAS 383,1385

Lodieu, N., Burningham, B., Hambly, N. C., \& Pinfield, D. J. 2009, MNRAS, 397,258

Lucas, P. W., Tinney, C. G., Burningham, B., et al. 2010, MNRAS, 408, L56

Mandel, H., Seifert, W., Hofmann, R., et al. 2008, SPIE, 7014, 124

Marocco, F., Smart, R. L., Jones, H. R. A., et al. 2010, A\&A, 524, A38

Pier, J. R., Munn, J. A., Hindsley, R. B., et al. 2003, AJ, 125, 1559

Pinfield, D. J., Burningham, B., Tamura, M, et al. 2008, MNRAS, 390, 304

Pinfield, D. J., Burningham, B., Lodieu, N., et al. 2012, MNRAS, 422, 1922

Scholz, R.-D. 2010, A\&A, 515, A92 (S10)

Scholz, R.-D., Bihain, G., Schnurr, O., \& Storm, J. 2011, A\&A, 532, L5 (S11)

Seifert, W., Ageorges, N., Lehmitz, M., et al. 2010, SPIE, 7735, 256

Skrutskie, M. F., Cutri, R. M., Stiening, R., et al. 2006, AJ, 131, 1163

Tinney, C. G., Burgasser, A. J., \& Kirkpatrick, J. D. 2003, AJ, 126, 975

Tokunaga, A. T., Simons, D. A., \& Vacca, W. D. 2002, PASP, 114, 180

Tsvetanov, Z. I., Golimowski, D. A., Zheng, W., et al. 2000, ApJ, 531, L61

Warren, S. J., Mortlock, D. J., Leggett, S. K., et al. 2007, MNRAS, 381, 1400

Wright, E. L., Eisenhardt, P. R. M., Mainzer, A. K., et al. 2010, AJ, 140, 1868

Pages 6 to 8 are available in the electronic edition of the journal at http: //www . aanda. org 


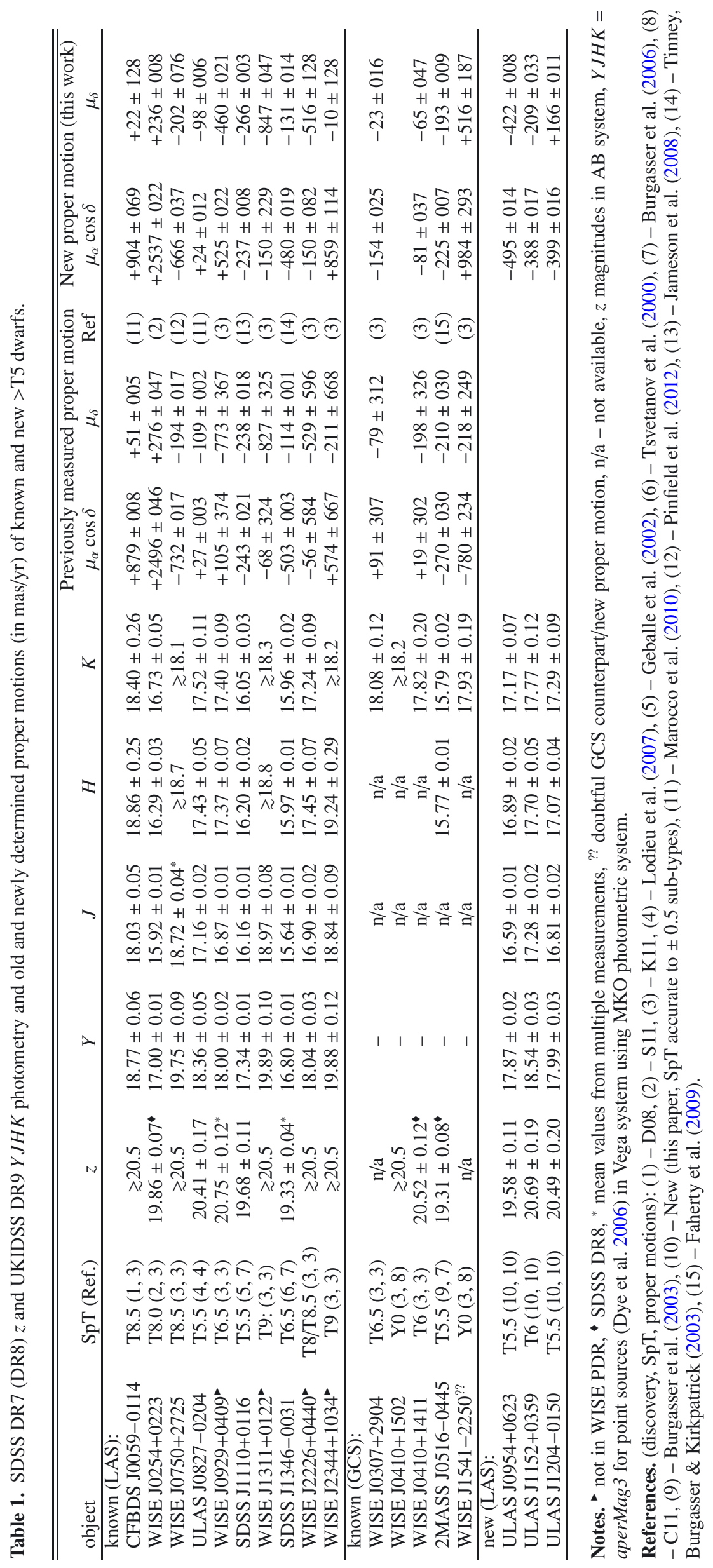


R.-D. Scholz et al.: UKIDSS detections of cool brown dwarfs $(R N)$

Table 2. $\alpha, \delta(\mathrm{J} 2000.0)$ of known > T5 dwarfs in UKIDSS LAS, etc.

\begin{tabular}{|c|c|c|c|c|}
\hline $\begin{array}{l}\text { Object } \\
\alpha \text { [deg] }\end{array}$ & $\delta[\mathrm{deg}]$ & Epoch & ID & Source \\
\hline $\begin{array}{l}\text { CFBDS J0059 } \\
14.7949709 \\
14.7949181 \\
14.7952080 \\
14.7952146 \\
\end{array}$ & $\begin{array}{l}-0114^{1}: \\
-01.2336947 \\
-01.2337060 \\
-01.2337464 \\
-01.2337521 \\
\end{array}$ & $\begin{array}{l}2005.732 \\
2005.732 \\
2006.885 \\
2006.885 \\
\end{array}$ & $\begin{array}{c}292702 \\
293033 \\
1581752 \\
1584033 \\
\end{array}$ & $\begin{array}{l}\text { ULAS } H \\
\text { ULAS } K \\
\text { ULAS } Y \\
\text { ULAS } J \\
\end{array}$ \\
\hline $\begin{array}{l}\text { WISE J0254+ } \\
43.5399358 \\
43.5399337 \\
43.5399295 \\
43.5399246 \\
\end{array}$ & $\begin{array}{l}223^{2,3}: \\
+02.3996412 \\
+02.3996466 \\
+02.3996464 \\
+02.3996529 \\
\end{array}$ & $\begin{array}{l}10.734 \\
10.734 \\
10.718 \\
10.718 \\
\end{array}$ & $\begin{array}{l}3859643 \\
3859715 \\
3840324 \\
3840484 \\
\end{array}$ & $\begin{array}{l}\text { ULAS } H \\
\text { ULAS } K \\
\text { ULAS } Y \\
\text { ULAS } J\end{array}$ \\
\hline $\begin{array}{l}\text { WISE J0750+ } \\
117.5164404 \\
117.5159819 \\
117.5159827\end{array}$ & $\begin{array}{l}725^{3}: \\
+27.4293472 \\
+27.4292434 \\
+27.4292398\end{array}$ & & & $\begin{array}{l}\text { ULAS } J \\
\text { ULAS } Y \\
\text { ULAS } J\end{array}$ \\
\hline $\begin{array}{l}\text { ULAS J0827- } \\
126.7819292 \\
126.781892 \\
126.7819523 \\
126.7819511 \\
126.7819424 \\
126.7819506 \\
\end{array}$ & $\begin{array}{l}204^{4}: \\
-02.0687722 \\
-02.068816 \\
-02.0689431 \\
-02.0689491 \\
-02.0689509 \\
-02.0689568 \\
\end{array}$ & & $\begin{array}{c}2259 \\
465119 \\
473125 \\
478440 \\
477757 \\
\end{array}$ & $\begin{array}{l}2 \mathrm{M} J^{\star} \\
\text { SDSS } \\
\text { ULAS } Y \\
\text { ULAS } J \\
\text { ULAS } H \\
\text { ULAS } K \\
\end{array}$ \\
\hline $\begin{array}{l}\text { WISE J0929+ } \\
142.276914 \\
142.2776989 \\
142.277670 \\
142.2777383 \\
142.2781424 \\
142.2781455 \\
\end{array}$ & $\begin{array}{c}+04.1663975 \\
+04.166407 \\
+04.1663741 \\
+04.1660432 \\
+04.1660397\end{array}$ & & $\begin{array}{c}2125 \\
1219425 \\
6749 \\
1399129 \\
3028681 \\
3029035 \\
\end{array}$ & $\begin{array}{l}\text { SDSS } \\
\text { ULAS } K \\
\text { SDSS } \\
\text { ULAS } H \\
\text { ULAS } J \\
\text { ULAS } Y \\
\end{array}$ \\
\hline $\begin{array}{l}\text { SDSS J1110+ } \\
167.541711 \\
167.541674 \\
167.5411353 \\
167.5411422 \\
167.5410610 \\
167.5410582\end{array}$ & $\begin{array}{c}+01.270302 \\
+01.270298 \\
+01.2697012 \\
+01.2697045 \\
+01.2695783 \\
+01.2695767\end{array}$ & $\begin{array}{l}2000.126 \\
2000.343 \\
2008.373 \\
2008.373 \\
2009.978 \\
2009.978\end{array}$ & $\begin{array}{c}1462 \\
2325845 \\
2325865 \\
3085143 \\
3085325\end{array}$ & $\begin{array}{c}\text { 2M } \\
\text { SDSS } \\
\text { ULAS } H \\
\text { ULAS } K \\
\text { ULAS } Y \\
\text { ULAS } J\end{array}$ \\
\hline & $\begin{array}{l}+01.3817322 \\
+01.3817386\end{array}$ & & & \\
\hline $\begin{array}{l}\text { SDSS J1346- } \\
206.693495 \\
206.693104 \\
206.692471 \\
206.6921079 \\
206.6921118 \\
206.6921010 \\
206.6921028 \\
206.6920776\end{array}$ & $\begin{array}{c}-00.530639 \\
-00.530592 \\
-00.530844 \\
-00.5309516 \\
-00.5309485 \\
-00.5309524 \\
-00.5309471 \\
-0.5309553\end{array}$ & $\begin{array}{l}1999.221 \\
2001.093 \\
2006.395 \\
2009.129 \\
2009.129 \\
2009.134 \\
2009.134 \\
2010.182\end{array}$ & $\begin{array}{c}756 \\
6166 \\
2369313 \\
2369436 \\
2350848 \\
2351038\end{array}$ & $\begin{array}{c}\text { SDSS } \\
2 \mathrm{M} \\
\text { SDSS } \\
\text { ULAS } H \\
\text { ULAS } K \\
\text { ULAS } Y \\
\text { ULAS } J \\
\text { WISE }\end{array}$ \\
\hline $\begin{array}{l}\text { WISE J2226+ } \\
336.5960939 \\
336.5961029 \\
336.5960903 \\
336.5960866 \\
\end{array}$ & $\begin{array}{l}+04.6678106 \\
+04.6678081 \\
+04.6678034 \\
+04.6677827 \\
\end{array}$ & $\begin{array}{l}2009.620 \\
2009.620 \\
2009.641 \\
2009.641 \\
\end{array}$ & $\begin{array}{l}2784734 \\
2783676 \\
2797400 \\
2797524 \\
\end{array}$ & $\begin{array}{l}\text { ULAS } Y \\
\text { ULAS } J \\
\text { ULAS } H \\
\text { ULAS } K\end{array}$ \\
\hline $\begin{array}{l}\text { WISE J2344+ } \\
356.1923904 \\
356.1924237 \\
356.1924059\end{array}$ & $\begin{array}{l}+10.5710685 \\
+10.5710277 \\
+10.5710051\end{array}$ & $\begin{array}{l}2009.668 \\
2009.668 \\
2009.827\end{array}$ & $\begin{array}{l}2823367 \\
2823532 \\
2944838\end{array}$ & $\begin{array}{l}\text { ULAS } Y \\
\text { ULAS } J \\
\text { ULAS } H\end{array}$ \\
\hline
\end{tabular}

Notes. ${ }^{1}$ - additional epoch in D08, ${ }^{2}$ - more data in S11 (SDSS DR8, 2MASS), L11 (Pan-STARRS1), ${ }^{3}$ - WISE and Spitzer epochs in K11, ${ }^{4}$ - seen in WISE $w 2$ image but no catalogue entry, ${ }^{\star}$ - our visual detection. IDs are run and multiframe numbers for SDSS and UKIDSS LAS (ULAS), respectively.
Table 3. $\alpha, \delta$ (J2000.0) of known > T5 dwarfs in UKIDSS GCS, etc.

\begin{tabular}{|c|c|c|c|c|}
\hline $\begin{array}{l}\text { Object } \\
\alpha \text { [deg] }\end{array}$ & $\delta[\mathrm{deg}]$ & Epoch & ID & Source \\
\hline $\begin{array}{l}\text { WISE J0307- } \\
46.8525439\end{array}$ & $\begin{array}{l}904^{1}: \\
+29.0798528\end{array}$ & 2008.786 & 2334900 & UGCS $K$ \\
\hline $\begin{array}{l}\text { WISE J0410- } \\
62.7270953 \\
62.7269979 \\
\end{array}$ & $\begin{array}{l}411^{1}: \\
+14.1920928 \\
+14.1921757\end{array}$ & $\begin{array}{l}2005.781 \\
2006.367 \\
\end{array}$ & $\begin{array}{c}322532 \\
6003 \\
\end{array}$ & $\begin{array}{c}\text { UGCS } K \\
\text { SDSS } \\
\end{array}$ \\
\hline $\begin{array}{l}\text { 2MASS J051 } \\
79.039410 \\
79.0392237 \\
79.0386926 \\
79.0386834 \\
79.0386734\end{array}$ & $\begin{array}{l}-0445: \\
-04.763872 \\
-04.7640431 \\
-04.7644903 \\
-04.7644923 \\
-04.7645655\end{array}$ & $\begin{array}{l}1998.721 \\
2000.934 \\
2009.893 \\
2009.893 \\
2010.182\end{array}$ & $\begin{array}{c}1927 \\
2998751 \\
2998937\end{array}$ & $\begin{array}{c}2 \mathrm{M} \\
\text { SDSS } \\
\text { UGCS } H \\
\text { UGCS } K \\
\text { WISE }\end{array}$ \\
\hline $\begin{array}{l}\text { WISE J1541- } \\
235.4642206\end{array}$ & $\begin{array}{l}250^{1}: \\
-22.8407768\end{array}$ & 2008.301 & 2323120 & UGCS $K^{? ?}$ \\
\hline
\end{tabular}

Notes. ${ }^{1}$ - additional WISE and Spitzer epochs in K11, ?? - doubtful counterpart. ID are run and multiframe numbers for SDSS DR8 and UKIDSS GCS (UGCS), respectively.

Table 4. Multi-epoch $\alpha, \delta$ (J2000.0) of new late-T dwarf candidates.

\begin{tabular}{|c|c|c|c|c|}
\hline $\begin{array}{l}\text { Object } \\
\alpha \text { [deg] }\end{array}$ & $\delta[\operatorname{deg}]$ & Epoch & ID & Source \\
\hline \multicolumn{5}{|c|}{ ULAS J0954+0623: } \\
\hline 148.625883 & +06.387122 & 2000.153 & & $2 \mathrm{M} J^{\star}$ \\
\hline 148.625654 & +06.386935 & 2002.174 & 3015 & SDSS \\
\hline 148.6249784 & +06.3863407 & 2007.214 & 1287023 & ULAS $K$ \\
\hline 148.6249374 & +06.3863301 & 2007.277 & 1390233 & ULAS $H$ \\
\hline 148.6246477 & +06.3860939 & 2009.332 & 2442500 & ULAS $J$ \\
\hline 148.6245730 & +06.3860068 & 2009.942 & 3052277 & ULAS $Y$ \\
\hline \multicolumn{5}{|c|}{ ULAS J1152+0359: } \\
\hline 178.124725 & +03.991431 & 2000.181 & & $2 \mathrm{M} J^{\star}$ \\
\hline 178.124063 & +03.991187 & 2006.023 & 5973 & SDSS \\
\hline 178.1238153 & +03.9910013 & 2008.375 & 2326168 & ULAS $H$ \\
\hline 178.1237922 & +03.9910487 & 2008.375 & 2326188 & ULAS $K$ \\
\hline 178.1236578 & +03.9909038 & 2009.978 & 3084746 & ULAS $Y$ \\
\hline 178.1236518 & +03.9908986 & 2009.978 & 3084839 & ULAS $J$ \\
\hline \multicolumn{5}{|c|}{ ULAS J1204-0150: } \\
\hline 181.187079 & -01.843475 & 1999.077 & & $2 \mathrm{M} J^{\star}$ \\
\hline 181.187242 & -01.843383 & 1999.077 & & $2 \mathrm{M} H^{\star}$ \\
\hline 181.187080 & -01.843431 & 2000.116 & 1140 & SDSS \\
\hline 181.1861442 & -01.8430247 & 2008.395 & 2327790 & ULAS $H$ \\
\hline 181.1861488 & -01.8430134 & 2008.395 & 2327810 & ULAS $K$ \\
\hline 181.1861335 & -01.8430218 & 2008.403 & 2329084 & ULAS $Y$ \\
\hline 181.1861363 & -01.8430238 & 2008.403 & 2329105 & ULAS $J$ \\
\hline
\end{tabular}

Notes. ID are run and multiframe numbers for SDSS and UKIDSS LAS (ULAS), respectively. ${ }^{\star}$ indicate our visual detections. 
A\&A 541, A163 (2012)

Table 5. Spectral indices and corresponding types and classification from comparison with templates for three new late-T dwarfs.

\begin{tabular}{lccc}
\hline \hline Index & ULAS J0954+0623 & ULAS J1152+0359 & ULAS J1204-0150 \\
\hline $\mathrm{H}_{2} \mathrm{O}-\mathrm{H}$ & $0.319 \pm 0.012(\mathrm{~T} 6 / \mathrm{T} 5)$ & $0.299 \pm 0.021(\mathrm{~T} 6)$ & $0.315 \pm 0.015(\mathrm{~T} 6 / \mathrm{T} 5)$ \\
$\mathrm{CH}_{4}-\mathrm{H}$ & $0.347 \pm 0.012(\mathrm{~T} 6 / \mathrm{T} 5)$ & $0.304 \pm 0.016(\mathrm{~T} 6)$ & $0.369 \pm 0.012(\mathrm{~T} 5 / \mathrm{T} 6)$ \\
$\mathrm{CH}_{4}-\mathrm{K}$ & $0.233 \pm 0.022(\mathrm{~T} 5 / \mathrm{T} 4)$ & $0.199 \pm 0.030(\mathrm{~T} 5)$ & $0.225 \pm 0.024(\mathrm{~T} 5)$ \\
$\mathrm{SpT}(\mathrm{J})$ & $\mathrm{T} 5.5$ & $\mathrm{~T} 6.5$ & $\mathrm{~T} 5.5$ \\
$\left.\mathrm{SpT}_{\mathrm{H}} \mathrm{HK}\right)$ & $\mathrm{T} 5.5$ & $\mathrm{~T} 6$ & $\mathrm{~T} 5.5$ \\
$\mathrm{SpT}_{\text {adopted }}$ & $\mathrm{T} 5.5$ & $\mathrm{~T} 6$ & $\mathrm{~T} 5.5$ \\
\hline
\end{tabular}

